\title{
Blueberry juice as a nutraceutical approach to prevent prediabetes progression in an animal model: focus on hepatic steatosis
}

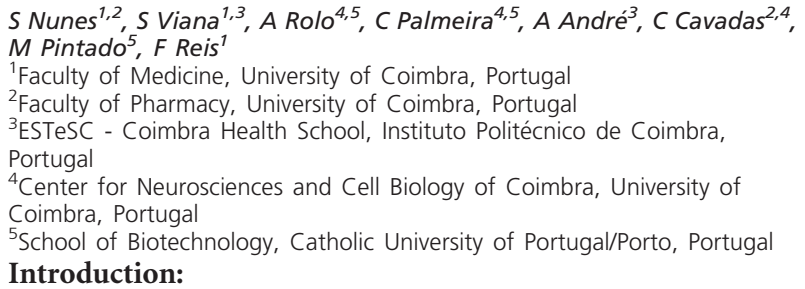

Blueberries (BB) consumption has been recognized as beneficial for metabolic disorders through its antioxidant, anti-inflammatory and insulin-sensitizing properties; however, eir effect against progression from prediabetes to diabetes remains unknown.

\section{Objectives:}

To assess the effects of blueberries juice (BJ) in a prediabetic animal model displaying hepatic steatosis.

\section{Methods:}

A rat model of evolutive prediabetes [Male Wistar rats, 8 weeks old] was developed by ingestion of a high-sucrose ( $\mathrm{HSu}, 35 \%$ ) diet for 9 weeks (W9), supplemented by a high-fat diet (HF, $60 \%$ ) for further 14 weeks (HSuHF, W23), vs control with standard diet. Half of the animals $(n=8$ /group) daily received BJ $(25 \mathrm{~g} / \mathrm{Kg} \mathrm{BW}$, orally) between W9 to W23. Glycemic, insulinemic and lipidic profiles were assessed, as well as markers of liver dysfunction/damage, including morphology, echogenicity, fibrosis/steatosis and mitochondrial function (ultrasonography, histochemical staining techniques and bioenergetics assays). ( $n=16$ /group Values are means \pm S.E.M. (ANOVA followed by post-hoc comparisons).Results: BJ treatment significantly $(\mathrm{p}<0.05$ or $\mathrm{p}<0.01)$ prevented the HSuHF-evoked metabolic and liver impairments, with amelioration of glucose tolerance, insulin sensitivity and hypertriglyceridemia, together with attenuation of liver damage, as seen by improved mitochondrial function and hepatic steatosis.

\section{Conclusions:}

This work provides novel evidence on BJ ability to prevent the aggravation of prediabetes induced by $\mathrm{HSuHF}$ diet with benefits against hepatic steatosis, although the precise mechanisms should be further explored. Bioactive compounds from $\mathrm{BB}$ are an exciting new area of food research and development of nutraceuticals that can be early used to prevent progression of prediabetes.

Support: CENTRO-01-0145-FEDER-000012-HealthyAging2020, FCT/COMPETE/FEDER (SFRH/BD/109017/2015; POCI-010145-FEDER-031712) and COAPE. 